\title{
Electrochemical synthesis of highly ordered magnetic multilayered nanowire arrays
}

\begin{abstract}
Electrochemical deposition is a versatile technique that has been employed to synthesize various types of one-dimensional nanostructures such as nanorods, nanotubes and nanowires to meet different requirements for applications. Magnetic nanowires in the form of multilayered structures, such as $\mathrm{Co} / \mathrm{Cu}$ and permalloy $(\mathrm{Ni80Fe} 20) / \mathrm{Cu}$, with ferromagnetic materials alternating with non-magnetic materials exhibit giant magnetoresistance (GMR) property that can be utilized in sensors and mass memory devices. This study focuses on the synthesis of highly ordered magnetic multilayered nanowire arrays using template-directed electrochemical deposition technique. The nanowires were electrodeposited within the nanopores of anodized alumina from sulphate baths via pulse potential technique. The structures and compositions of the wires were characterized using various microscopy and probe-based techniques. Magnetoresistance measurement was performed on the multilayered nanowire arrays.
\end{abstract}

Keyword: Giant magnetoresistance; Multilayered nanowires; Template-directed growth 